\title{
Research on Magnetic Property of Nd2Fe14B/a-Fe Nanocomposite Under Different Roller Speeds
}

\author{
Maoyuan Liu ${ }^{1}$ and Lei Chen ${ }^{*}, 2$ \\ ${ }^{1}$ College of Science of Tibet University, Lhasa City, Tibet Autonomous Region 850000, China \\ ${ }^{2}$ Haibin College of Beijing Jiaotong University, Huanghua City 061100, China
}

\begin{abstract}
Nano-composite permanent magnetic material is a new type of permanent magnetic material, and it is the synthesis of soft and hard magnetic phase within the nanoscale. On the basis of exchange coupling hard magnetizing theory, nano-composite permanent magnetic material can, at the same time, have high residual magnetization intensity of soft magnetic phase and high coercivity of hard magnetic phase, which can be developed into new generation high performance permanent magnetic material. Nevertheless, magnetic energy of permanent nano-composite magnet derived from experiments differs greatly from the theoretical value, and this is mainly due to fairly great difference between the micro-structure of material and the theoretical model. In this paper, the constituent was taken as $(\mathrm{Nd}, \mathrm{Pr}, \mathrm{Dy}) 2(\mathrm{Fe}$, $\mathrm{Nb}) 14 \mathrm{~B} / \alpha-\mathrm{Fe}$, and the fusant rapid quenching method was adopted to study the impact of different roller speeds on the magnetic property. Moreover, through the result of VSM, XRD and SPM, the magnetic property, phase composition and micro structure of alloy were analyzed.
\end{abstract}

Keywords: Fusant quick quenching, magnetic property, nano-composite permanent magnetic material.

\section{INTRODUCTION}

$\mathrm{Nd} 2 \mathrm{Fe} 14 \mathrm{~B} / \mathrm{\alpha}-\mathrm{Fe}$, a nano-composite permanent magnetic material has good magnetic property and very great development potential, and thus draws wide attention. Nevertheless, during long term research, it has been found that this material also has some disadvantages and whose partial magnetic property can be improved via mixing method. In this paper, $\mathrm{Nd} 2 \mathrm{Fe} 14 \mathrm{~B} / \alpha-\mathrm{Fe}$ nano-composite material was adopted as the base to add in the elements to optimize magnetic property. For the selection of substitute element, both $\mathrm{Pr} 2 \mathrm{Fe} 14 \mathrm{~B}$ and Dy2Fe14B have anisotropy field higher than $\mathrm{Nd} 2 \mathrm{Fe} 14 \mathrm{~B}$ [1], in which, the addition of $\mathrm{Nb}$ can refine the crystal grain and enhance the exchange coupling effect. $\mathrm{Nd}$ is substituted by Pr and Dy to improve the anisotropy field of composite magnet, with small quantity of $\mathrm{Nb}$ substitutes $\mathrm{Fe}$ to refine the crystal grain structure and enhance exchange coupling effect [2] of hard magnetic phase Nd2Fe14B and soft magnetic phase $\alpha-\mathrm{Fe}$.

Due to the above consideration, in this paper the constituent of set sample was (Nd, Pr, Dy) $2(\mathrm{Fe}, \mathrm{Nb}) 14 \mathrm{~B} / \mathrm{\alpha}-$ Fe. Furthermore, according to documentation [3], the content of $\alpha-\mathrm{Fe}$ was determined up to $25.9 \%$, and the detailed mix ratio is shown in Table $\mathbf{1}$.

\section{EXPERIMENT}

At first, the raw material was prepared by $100 \mathrm{~g}$ via designed proportion, and then it was placed into water-

*Address correspondence to this author at the Haibin College of Beijing Jiaotong University, Huanghua City, 061100, China; Tel: +8613821392810; E-mail: chenlei@163.com cooled copper crucible of electric arc furnace. Prior to smelting, it was vacuumized to below $10 \mathrm{~Pa}$ via mechanical pump, and about $4.5 \times 10-4 \mathrm{~Pa}$ via molecular pump. Following this, the high purity argon was filled in for protection, while after arc strike was observed by high voltage current, smelting was started with a current of 500 A. During smelting, the arc torch temperature can be set up to 1500 . Smelting was repeated 3-4 times, till the sample was made even. After smelting, the sample was taken out after it was cooled down.

Table 1. Design of constituents of experiment samples.

\begin{tabular}{|c|c|}
\hline$\alpha \alpha$-Fe Content & Sample \\
\hline \hline $6.0 \%$ & $\mathrm{Nd} 5.4 \mathrm{Pr} 4.6 \mathrm{Dy} 1 \mathrm{Fe} 82 \mathrm{Nb} 1 \mathrm{~B} 6 \mathrm{x}$ \\
\hline $17.9 \%$ & $\mathrm{Nd} 4.5 \mathrm{Pr} 4.2 \mathrm{Dy} 0.8 \mathrm{Fe} 84 \mathrm{Nb} 1 \mathrm{~B} 5 \mathrm{x}$ \\
\hline $25.9 \%$ & $\mathrm{Nd} 4.2 \mathrm{Pr} 3.6 \mathrm{Dy} 0.7 \mathrm{Fe} 85.5 \mathrm{Nb} 1 \mathrm{~B} 5$ \\
\hline
\end{tabular}

The ingot casting was broken into 10-20 g small pieces, and placed into high temperature resistance quartz tube with $0.5 \mathrm{~mm}$ diameter small hole at the lower end. The quartz tube was placed in the vacuum quickly quenching chamber, which was vacuumized to nearly $4.5 \times 10-4 \quad \mathrm{~Pa}$ via mechanical pump and molecular pump. The sample was heated and molten by the vortex current, with the melting temperature being 1300 . The molten sample was sprayed on the surface of cooling roller via argon pressure of 0.5-1.2 atm, and the speed of cooling roller was set as $10-45 \mathrm{~m} / \mathrm{s}$. The thickness of the thin strip was about $20-40 \mu \mathrm{m}$. For the sample made at high quick quenching speed, being an amorphous structure, the amorphous phase during crystallization should involve appropriate heat treatment, so 
that $(\mathrm{Nd}, \mathrm{Pr}, \mathrm{Dy}) 2(\mathrm{Fe}, \mathrm{Nb}) 14 \mathrm{~B} / \alpha-\mathrm{Fe}$ nano-composite structure can be formed.

The sample thin strip was placed in the sample tray made from the thin iron piece, and then placed in the tube furnace passing through the argon current to conduct crystallization treatment on the sample via controlling the increasing rate of temperature, heating temperature and heating time. After crystallization, the sample was taken away from the heating furnace and rapidly cooled down by water to derive the series of thin strips sample.

\section{PHASE STRUCTURE ANALYSIS}

Fig. (1) shows the XRD spectrum of sample with $25.9 \%$ $\alpha-\mathrm{Fe}$ constituent at different roller speeds, and it shows that at any speed, $\alpha-\mathrm{Fe}$ and $\mathrm{Nd} 2 \mathrm{Fe} 14 \mathrm{~B}$ phase can be observed, showing that the thin strip is double phase composite material. With increasing roller speed, the thin strip sample is gradually changed to the amorphous structure from the crystal structure. At $10 \mathrm{~m} / \mathrm{s}$, the sample has very strong characteristics of crystal and sharp diffraction peak, and according to Scherrer Equation [4], the crystal size at this status is fairly great. The strength of (004) and (008) diffraction peak is fairly great, and this means that inside the sample, the hard magnetic phase crystal grows along the vertical direction to the thin strip face, and the easy magnetization axis is vertical to the thin strip face. With the increase in the roller speed, the strength of (004) and (008) diffraction peak is gradually decreased, with increase in the strength of (214) and (410) diffraction peak, while the other $2: 14: 1$ phase characteristic peak occurs at the circumference of (214) diffraction peak. This means that the crystal grain of 2:14:1 phase grows by approaching the direction parallel to the thin strip face, and the easy magnetization axis is changed to the direction parallel to the thin strip face. At 30 $\mathrm{m} / \mathrm{s}$, the diffraction peak is widened, and the increase of half of the width means that crystal grain size is decreased inside the material with increase in the exchange coupling effect. Furthermore, there are many diffraction peaks; this means that the internal texture is enhanced with the advantage of improving the magnetic property. With increase in the quick quenching speed, the amorphous structure is obtained and the sample achieves over-quick quenching status.

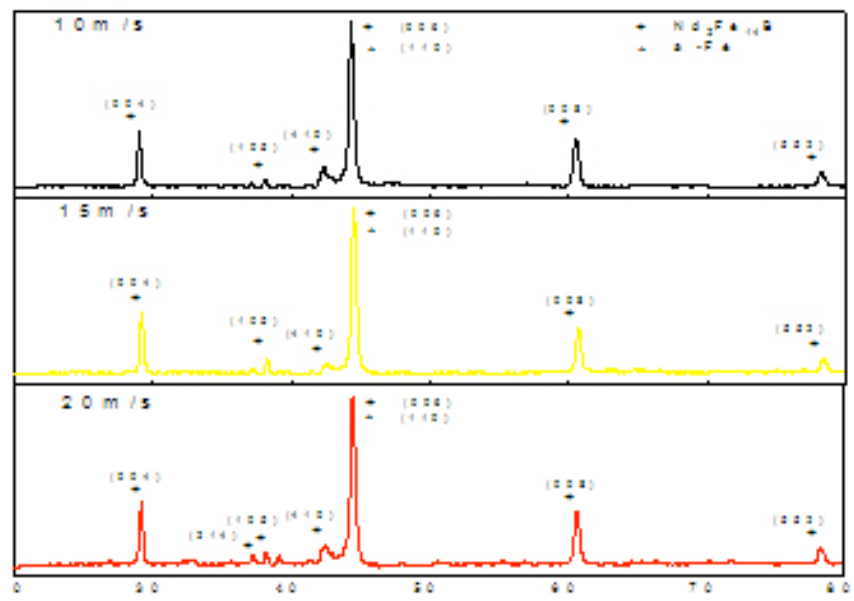

Research by Zhang [5] et al. deems that, at fairly low quick quenching speed, easy magnetization axis of thin strip is gradually changed into a direction parallel to the thin strip plane. Because, on one hand, when the alloy of molten status is sprayed on the roller face, since there is a great difference of temperature in the roller-fit face and free face, the incur temperature is observed inside the thin strip gradient vertical to the roller face, i.e., the direction of heat current is vertical to the roller face. According to the theory, on the basis of temperature gradient, quick quenching thin strip grows on the moving roller face, due to tensile stress tangential to the roller. Crystal grain has slippage, and is rotated by anisotropy of strain energy. At joint effect of tensile stress and strain energy, easy magnetization axis of RE2Fe14B can be easily distributed at the direction vertical to the thin strip. On the other hand, coring and growth of double phase nanocomposite permanent magnetic material rely on the diffusion extent of the atom, and during quick quenching process with fairly high roller speed, coring and growth of RE2Fe14B phase are fairly difficult. Nevertheless, crystallization temperature of $\alpha$-Fe phase is fairly low, and can be firstly separated out during this process to form (110) texture. Thus, $\alpha$-Fe can provide crystallization core during the growth process of RE2Fe14B phase, so that the crystal face of RE2Fe14B crystal grain can have the priority of growth at the induction of (110) texture of $\alpha-F e$. Moreover, the direction of easy magnetization of crystal grain of $\mathrm{RE} 2 \mathrm{Fe} 14 \mathrm{~B}$ phase is parallel to the thin strip plane, and builtup magnet has anisotropy.

\section{SPM ANALYSIS}

Fig. (2) is the atomic force microphotograph (AFM) at quick quenching speed of $20 \mathrm{~m} / \mathrm{s}$ and $25 \mathrm{~m} / \mathrm{s}$, with scan range of $2 \mu \mathrm{m} \times 2 \mu \mathrm{m}$. It shows that, the size of the crystal grain size is in the range of nanometers and is less than 100 $\mathrm{nm}$. With increase in the roller speed, mostly of them are $60 \sim 90 \mathrm{~nm}$, and there is a great difference in the size of crystal grains. Evenness in the distribution is therefore fairly bad; at $25 \mathrm{~m} / \mathrm{s}$, the crystal grain distribution is at $40 \sim 60 \mathrm{~nm}$ and is relatively concentrated, which significantly improves the magnetic property. According to the existing theory, fine size and even distribution of crystal grain are in favor of improving the exchange coupling effect. Fairly, the strong

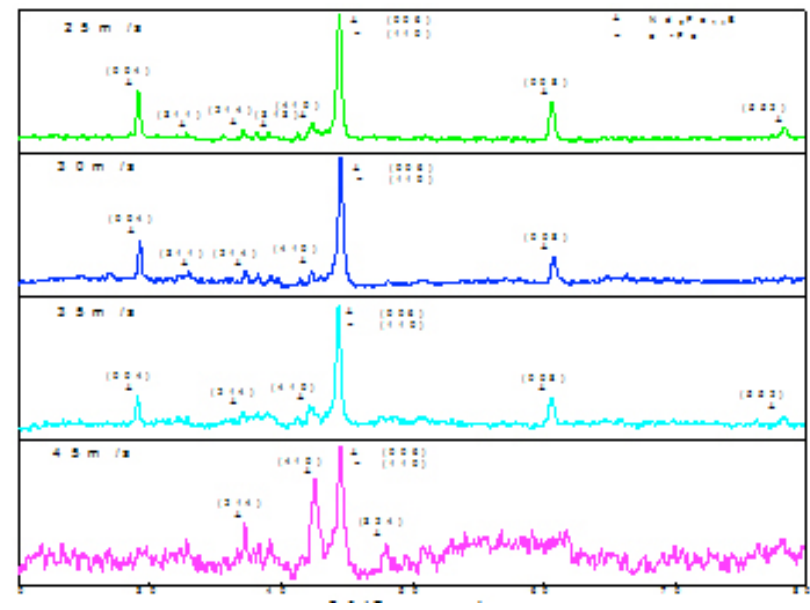

Fig. (1). XRD spectrum of sample of $25.9 \% \alpha-\mathrm{Fe}$ content at different roller speeds. 
exchange coupling effect can enable the magnetic moment of crystal grain rotate toward the same direction to improve the residual magnetism of nano-composite magnet. Furthermore, fine and even crystal grains are comprehensively coupled in favor of effective anisotropy of the soft magnetic phase of double phase composite material, so as to improve the coercivity of magnet. Magnetic energy product of magnet is determined by the product of residual magnetism and coercivity, within the certain range. The magnetic property of quick quenching thin strip is increased by increasing the roller speed.
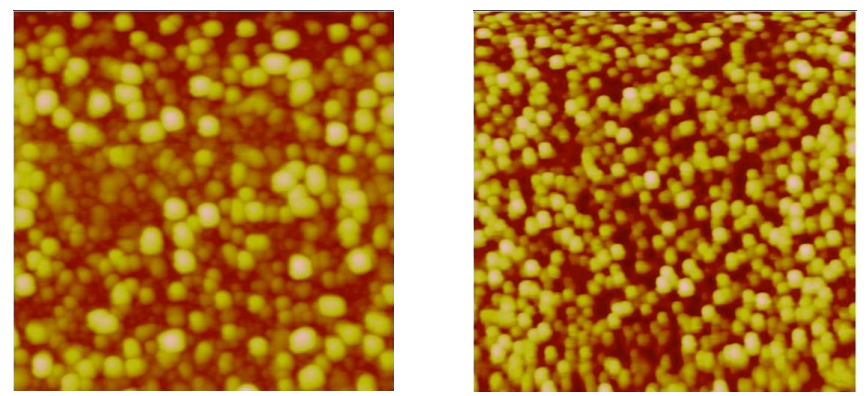

Fig. (2). Comparison $(2 \mu m \times 2 \mu m)$ on AFM chart of sample of $25.9 \% \alpha-F e$ content at quick quenching speed of $20 \mathrm{~m} / \mathrm{s}$ (left) and $25 \mathrm{~m} / \mathrm{s}$ (right).

\section{COMPARISON ON MAGNETIC PROPERTY}

This section discusses the magnetic property of the sample of $25.9 \% \alpha$-Fe content at different quick quenching speeds. The external field applied by VSM is 1T.

Table 2. Magnetic property parameters of thin strip at different quick quenching speeds.

\begin{tabular}{|c|c|c|c|c|}
\hline $\mathbf{v}(\mathbf{m} / \mathbf{s})$ & Hci $(\mathbf{k A} / \mathbf{m})$ & $\operatorname{Br}(\mathbf{T})$ & $\mathbf{M r} / \mathbf{M s}$ & $\mathbf{( B H )} \mathbf{M a x}(\mathbf{k J} / \mathbf{m 3})$ \\
\hline \hline 10 & 141.6 & 0.47 & 0.51 & 10.53 \\
\hline 15 & 177.7 & 0.49 & 0.56 & 14.9 \\
\hline 20 & 206.5 & 0.56 & 0.58 & 16.64 \\
\hline 25 & 409.7 & 0.7 & 0.69 & 41.05 \\
\hline 30 & 538.3 & 0.68 & 0.73 & 53.44 \\
\hline 35 & 517 & 0.45 & 0.73 & 27.19 \\
\hline 45 & 488.4 & 0.43 & 0.71 & 13.67 \\
\hline
\end{tabular}

Table 2 shows that for nano-composite material with $\mathrm{Nd} 4.2 \mathrm{Pr} 3.6 \mathrm{Dy} 0.7 \mathrm{Fe} 85.5 \mathrm{Nb} 1 \mathrm{~B} 5$ constituent, the parameter of magnetic property is greatly impacted by quick quenching speed. At $10 \mathrm{~m} / \mathrm{s}$, the thin strip has crystallization status. According to the XRD analysis, thick crystal grain's size is not in favor of exchange coupling effect, as its magnetic property is not good, since each parameter of magnetic property is not high. With increasing quick quenching speed, the XRD chart shows that the diffraction peak is widened, with increased quantity and enhanced internal texture. Furthermore, the SPM chart shows that, the crystal grain size gradually becomes small, with enhanced exchange coupling effect due to heteromorphism observed at one domain. At $30 \mathrm{~m} / \mathrm{s}$, the magnetic property was optimal:
$\mathrm{Hcj}=538.33 \mathrm{kA} / \mathrm{m}, \mathrm{Br}=0.68 \mathrm{~T}, \mathrm{Mr} / \mathrm{Ms}=0.73$, $(\mathrm{BH}) \max =$ $53.44 \mathrm{~kJ} / \mathrm{m} 3$. After quick quenching speed became greater than $30 \mathrm{~m} / \mathrm{s}$, the sample thin strip had better quick quenching status. The XRD analysis showed that, the thin strip had amorphous phase incurring decrease in the magnetic property. Coercivity, remanence ratio and magnetic energy product reached maximum. At $30 \mathrm{~m} / \mathrm{s}$, the residual magnetism reached maximum, while at $25 \mathrm{~m} / \mathrm{s}$, its value became $0.7 \mathrm{~T}$.

Magnetic property greatly changes with variation in the quick quenching speed, because the cooling speed of thin strip is different at setting process. When quick quenching speed was low, the cooling speed was also low at the setting process of thin strip, while when the super-cooling degree of fusant was relatively small, the coring rate was also fairly low, thus suggesting large size of the crystal grain size incurring uneven distribution of crystal grain, due to which, the time alloy thin strip became thick. When the quick quenching speed was $30 \mathrm{~m} / \mathrm{s}$, the mean thickness of the thin strip and the crystal grain was small, because the range of cooling speed and variation in accordance with the thickness were moderate. The degree of super-cooling increased to some extent, where the liquid alloy had very high coring rate which only ensured the thickness, so that the alloy can have a large number of even coring, and the growth thereof shall not go too far, to keep the crystal grain size small and texture even. It is approaching to microstructure of onedimensional theoretical model by assuming that the maximum exchange coupling effect was acquired to prove fairly good exchange coupling effect between the soft and hard magnetic phase. (AUTHOR: The highlighted is vague and can be re-phrased) When quick quenching speed was too high, the super-cooling fusant temperature was very low. According to the coring theory [6], the diffusion rate of atom in fusant and related energy fluctuation and concentration fluctuation apparently decreased, furthermore, with increase of setting cooling speed, coring time shall also be shortened, and these factors shall counteract the impact of increase of drive of thermo-dynamics and coring rate shall apparently decrease. When coring rate is approaching to 0 , atom configuration in the fusant shall basically be invariable, i.e., during setting process it is "frozen" to form the long range and unordered amorphous status, and suppress the formation of crystal status. (AUTHOR: The highlighted is vague and must be re-phrased)

According to the traditional Stoner-Wohlfarth ferromagnetic theoretical model [7], the maximum theoretical value of remanence ratio of isotropy with permanent magnet of single easy axis was only $0.5 \mathrm{Ms}$. In the nano-composite permanent magnetic material, the remanence ratio was $\mathrm{Mr} / \mathrm{Ms}>0.5$, i.e., showing aresidual magnetism enhancement effect. Because the size of crystal grain of hard and soft magnetic phase was in the range of nanometer, in case of the effect of external magnetic field with the magnetic exchange coupling effect, the soft magnetic phase magnetic moved to the direction parallel to the hard magnetic phase moment, i.e., there was a strong ferromagnetic exchange coupling effect between the two phases. Therefore, magnetization and back magnetization of the permanent magnet showed the characteristics of single hard magnetic phase. The result shows that, there was a strong exchange coupling effect between the hard magnetic 
crystal grain and soft magnetic crystal grain in the prepared sample.

\section{BRIEF SUMMARY}

Magnetic property of the sample of constituent of $\mathrm{Nd} 4.2 \mathrm{Pr} 3.6 \mathrm{Dy} 0.7 \mathrm{Fe} 85.5 \mathrm{Nb} 1 \mathrm{~B} 5$ at different roller speeds was analyzed results of which are as follows:

(1) The XRD spectrum of thin strip at different quick quenching speeds was analyzed, along with the phase constituent of composite material subjected to the variation in each diffraction peak, to study the microstructure. At $30 \mathrm{~m} / \mathrm{s}$, there were many diffraction peaks with half peak width, suggesting strong internal texture of the magnet, small size of the crystal grain, and strong exchange coupling effect.

(2) The AFM image of thin strip with $20 \mathrm{~m} / \mathrm{s}, 25 \mathrm{~m} / \mathrm{s}$, and $30 \mathrm{~m} / \mathrm{s}$ was analyzed. Within the scan range of 2 $\mu \mathrm{m} \times 2 \mu \mathrm{m}$, the size of the crystal grain gradually became small and even with the high quick quenching speed. The fine and even crystal grain size proved to be effective for the exchange coupling effect. MFM chart of $30 \mathrm{~m} / \mathrm{s}$ shows that, there was heteromorphism in one domain due to strong exchange coupling effect.

(3) When $1 \mathrm{~T}$ external magnetic field was applied, the magnetic property of thin strip increased with increased quick quenching speed. It reached the maximum at $30 \mathrm{~m} / \mathrm{s}$ and then gradually decreased. The optimal magnetic property was: Hcj $=538.33$
$\mathrm{kA} / \mathrm{m}, \mathrm{Br}=0.68 \mathrm{~T}, \mathrm{Mr} / \mathrm{Ms}=0.73,(\mathrm{BH}) \quad \max =53.44$ $\mathrm{kJ} / \mathrm{m} 3$.

\section{CONFLICT OF INTEREST}

The authors confirm that this article content has no conflict of interest.

\section{ACKNOWLEDGEMENTS}

This work was supported by the Natural Science Foundation of China Tibet (Z2012A06G06/00).

\section{REFERENCES}

[1] Y. M. Zhu, S. Yang, and X. P. Song, "Impact to double phase $\mathrm{NdFeB}$ magnetic property by adding Tb", Journal of Chinese Electron Microscopy Society, vol. 24, no. 4, pp. 338, 2005.

[2] Z. M. Chen, Y. Q. Wu, and M. J. Kramer, "A study on the role of $\mathrm{Nb}$ in melt-spun nanocrystalline Nd-Fe-B magnets", J Magn Magn Mater, vol. 268, pp. 105-113, 2004.

[3] J. Bauer, M. Seeger, and H. Kronmuller, "Nanocrystalline FeNdB magnets with enhanced remanence", J Appl Phys, vol. 80, pp. 1667-1673, 1996.

[4] B. D. Cullity, Element of X-ray Diffraction. Addision-Wesley Reading MA, 1987.

[5] X. Y. Zhang, Y. Guan, and L. Yang, "Crystallographic texture and magnetic anisotropy of $\alpha-\mathrm{Fe} / \mathrm{Nd}_{2} \mathrm{Fe}_{14} \mathrm{~B}$ nanocomposites prepared by controlled melt spinning", Appl Phys Lett, vol. 79, no. 15, pp. 2426, 2001.

[6] T. Y. Cheng and S. H. Zhang, Quick setting technology and new type alloy. Beijing: Beijing Astronautic Publishing House, 1996.

[7] E. C. Stoner and E. P. Wohlfarth, "A mechanism of magnetic hysteresis in heterogeneous alloy", Philos Trans R Soc, vol. 240, pp. 599-602, 1948.

This is an open access article licensed under the terms of the Creative Commons Attribution Non-Commercial License (http://creativecommons.org/licenses/ by-nc/3.0/) which permits unrestricted, non-commercial use, distribution and reproduction in any medium, provided the work is properly cited. 